\title{
P I 6- I 2. Relative dominance of Gag-specific cytotoxic T lymphocytes is associated with viral load inversely in HIV-I clade B' infected Chinese
}

\author{
M Jia*1, J Chen¹, K Hong1, S Liu'1, X Zhang1, H Zhao1, M Altfeld², B Walker², \\ $\mathrm{X} \mathrm{Yu}{ }^{2}$ and $\mathrm{YShao}^{1}$
}

\author{
Address: ${ }^{1}$ Division of Virology and Immunology, National Center for AIDS/STD Control and Prevention, China CDC, Beijing, PR China and \\ 2Ragon Institute of MGH, MIT and Harvard, Boston, MA, USA \\ * Corresponding author
}

from AIDS Vaccine 2009

Paris, France. 19-22 October 2009

Published: 22 October 2009

Retrovirology 2009, 6(Suppl 3):P24 I doi:10.I 186/I742-4690-6-S3-P24I

This abstract is available from: http://www.retrovirology.com/content/6/S3/P24 I

(c) 2009 jia et al; licensee BioMed Central Ltd.

\section{Background}

The role of CD8 ${ }^{+} \mathrm{T}$ cells with cytotoxic (CTL) activity of different HIV proteins in controlling HIV-1 infection is still controversial, though a number of studies have suggested that gag-specific CTLs could play a superior role in viral control. The characterization of HIV-1-specific CTLs in genetic diverse individuals infected with locally prevalent HIV-1 strains will provide useful information in elucidating the mechanism of HIV-1 pathogenesis.

\section{Methods}

The HIV-1-specific CTLs were measured with an IFN- $\gamma$ ELISPOT assay by using overlapping peptides covering the whole consensus clades $\mathrm{B}$ proteome in 114 untreated HIV-1 clade B' infected Chinese. The correlation of CTL responses with immune control of HIV-1 infection was analyzed.

\section{Results}

The mean spot-forming cells $/ 10^{6}$ PBMCs of positive responses to each of the HIV-1 proteins were as follows: Gag, 2853; Pol, 1305; Env, 928; Nef, 1244; Tat, 543; Rev, 827; Vpr, 260; Vpu, 476 and Vif, 365. PBMC from 101/ $114(88.60 \%)$ subjects recognized at least one overlapping Gag peptide. Pol, Env, Nef, Tat, Rev, Vpr, Vpu and Vif were targeted by 85.09, 74.56, 78.95, 29.82, 29.82, 19.30, 12.28 and $32.46 \%$ of studied individuals, respectively. When viral loads were compared to the proportion of pro- tein-specific CTL responses of the total virus-specific responses, we found an inverse association between viral loads and the breadth $(\mathrm{p}<0.001)$ and magnitude $(\mathrm{p}<$ 0.001 ) of the relative Gag response, and a direct association between viral loads and the breadth of the relative $\operatorname{Tat}(\mathrm{p}=0.029), \operatorname{Pol}(\mathrm{p}=0.003)$ and $\operatorname{Env}(\mathrm{p}=0.029)$ response and magnitude of the relative Tat $(\mathrm{p}=0.043)$, $\operatorname{Pol}(\mathrm{p}=0.003)$ and $\operatorname{Env}(\mathrm{p}=0.030)$ response.

\section{Conclusion}

In present study, Gag is the most immunodominant region. The proportion of Gag-specific CTL responses among the total virus-specific CTL activity is inversely associated with viral loads. The dominance of Gag-specific responses may be an indicator of relative control of HIV infection. 